\title{
Sleep disturbance in obsessive compulsion disorder (OCD)
}

\begin{abstract}
Obsessive Compulsive Disorder (OCD) is now thought to be accompanied by sleep disorder. Some conclude that the thoughts and behaviors associated with OCD could become disruptive to sleep. Others have found a sleep disturbance characterized as late sleep onset in a significant number of OCD patients with reduced High Frequency (HF) Heart Rate Variability (HRV)! They acknowledge a relationship between cognitive inhibition and HF HRV in a sample of OCD patients. This supported the anticipated relationship between the role of executive functioning in the prefrontal cortex and vagally mediated HRV, indicating that relationship is also meaningful in OCD. A study in Norway found sleep disturbance characterized as late sleep onset in a significant number of OCD patients with reduced High Frequency (HF) Heart Rate Variability (HRV). This indicates an imbalance of the autonomic nervous system (dysautonomia). A study by Hinds et al. ${ }^{5}$ (OCD), individuals feel compelled to repeatedly perform security-related behaviors. (1) a starting problem - in which the level of excitation in response to stimuli suggesting potential danger is abnormally strong; versus (2) a stopping problem - in which the satiety-like process for shutting down securityrelated thoughts and actions is weak. By addressing sleep disturbance in OCD patients may ensure a holistic approach to treatment, enhance treatment efficacy, mitigate relapse and protect against the onset of co-morbid psychiatric illnesses.
\end{abstract}

\section{What is OCD and why is this important?}

Obsessive-compulsive disorder (OCD) is one of the anxiety disorders that may develop early in life. It may affect children, especially boys who also have a diagnosis of attention deficit hyperactivity disorder (ADHD). It is also often diagnosed through adolescence and into early adulthood. Among adults, it affects women and men equally. It occurs in $0.5-1 \%$ of people in a given year. OCD is characterized by two features: obsessions and compulsions. Obsessions in OCDs are persistent thoughts, images, or impulses that seem intrusive or inappropriate by others. These ideas are often associated with anxiety or distress. One common obsession relates to cleanliness and a belief that objects are contaminated with germs. Other compulsive thoughts in people with OCD are that they think that a task was not completed or done inappropriately. For example, there may be anxiety about turning off the stove or locking the door, even though these were in fact safely secured. These obsessions are often ignored or suppressed by engaging in some other thought or action, which is called a compulsion.

Compulsions are characterized as repetitive and deliberate actions that are done as a response to a specific obsession. It is reasoned that by performing these compulsive acts, the obsessive thoughts can be temporarily suppressed. Such compulsions are performed in a very predictable and ritualized fashion. Many have compulsions have a superstitious quality or have religious significance such as bowing or crossing oneself multiple times. In order to relieve anxiety about a fear of germs or sinful thought and, cleaning may occur. To verify that the door is in fact locked, it may be checked three times. Common compulsions include: Hand washing, checking, ordering, counting, praying, repeating words or tapping. These compulsions may provide transient relief, but soon the anxiety returns again and the compulsive act must be repeated. When these actions occupy more than 1 hour per day and interfere with the normal routine of life, OCD is diagnosed. ${ }^{1}$

\section{How does OCD affect sleep and cause insomnia?}

Some conclude that the thoughts and behaviors associated with
Volume 3 Issue I - 2019

\section{Trent W Nichols}

Quietmind Foundation, USA

Correspondence: Trent W Nichols, CNDD, 72I Ash Dr. Hanover PA 17331.717-633-5540 and Quietmind Foundation, Quietmind Foundation, Suite 315, 8I 20 Old York Rd. Elkins Park PAI 9027, Tel +610-940-0488, Fax 216-359-0630,

Email twnicholpa@comcast.net

Received: October 13, 2018 | Published: March 29, 2019
OCD could become disruptive to sleep. Accordingly, if you try to fall asleep at night but are constantly thinking about the things you need to check, you may have resulting insomnia. In fact, checking behaviors may be more disruptive if they involve leaving your sleep environment to reassure yourself. A study in Bergan Norway by Havnen and associates in Bergan Norway found sleep disturbance characterized as late sleep onset in a significant number of OCD patients with reduced High Frequency (HF) Heart Rate Variability (HRV)! A sample of 31 OCD patients was recruited from a waiting list. The OCD patients were characterized by a reduced HF heart variability only in a standing position. A majority of the sample reported high rates of sleep disturbances, which were significantly associated with symptoms of depression and anxiety but unrelated to HRV and OCD symptoms. The authors concluded that the study was to their knowledge the first to indicate a relationship between cognitive inhibition and HF HRV in a sample of OCD patients. They stated that metaphorically speaking, primary insomnia might be the consequence of a stuck gas pedal (arousal) but rather a result of impaired brakes (inhibition). They cited that the relationship between HRV and cognitive inhibition supported the anticipated relationship between the role of executive functioning in the prefrontal cortex and vagally mediated $\mathrm{HRV}$, indicating that relationship is also meaningful in OCD. ${ }^{2}$

Some research suggests that there may be less total sleep or more sleep disruption, but this has not been consistently demonstrated. It may not solely be due to the OCD but instead could relate to depression, which often coexists with it. More research is needed into the possible relationship between OCD and sleep disturbances. The Brain's Inability to See That Something is Safe, Causes OCD; Claire Wilson in a new report in New Scientist states, the repeated thoughts and urges of obsessive compulsive disorder (OCD) may be caused by an inability to learn to distinguish between safe and risky situations. She reports that focused therapy for OCD that is known as Exposure Response Prevention (ERP) therapy which involves patients trying to experience their triggers without doing their accompanying rituals such as touching a toilet seat without washing their hands afterwards -teaches the subject with OCD that nothing bad happens. Fineberg 
stated new findings may explain why people with OCD find this approach so difficult and it can take so long. "The bit of their brain that should be telling them it's safe isn't working. Now we can say to them this is why it's taking so long and we should stick with it." ${ }^{3}$ According to a study by Hinds et al. ${ }^{4}$ in obsessive-compulsive disorder (OCD), individuals feel compelled to repeatedly perform securityrelated behaviors, even though these behaviors seem excessive and unwarranted to them. They reasoned (1) a starting problem - in which the level of excitation in response to stimuli suggesting potential danger is abnormally strong; versus (2) astopping problem - in which the satiety-like process for shutting down security-related thoughts and actions is abnormally weak. The intensity of activation of security motivation was measured objectively by change in respiratory sinus arrhythmia using heart rate variability. ${ }^{4}$

\section{Brain genes in obsessive compulsion disorder}

A new study at the Broad Institute sequenced coding and regulatory elements for 608 genes potentially involved in obsessivecompulsive disorder in human, dog, and mouse. Using a new method that prioritizes likely functional variants, they found four strongly associated genes, validated in a larger cohort. Their findings suggest synaptic adhesion as a key component in compulsive behaviors. Since $\mathrm{OCD}$ is a severe psychiatric disorder also linked to abnormalities in glutamate signaling and the cortico-striatal circuit, the psychiatric states may result from an imbalance of excitatory glutamate and inhibitory GABAergic neuron differentiation, a process that involves the genes NRXN1 a synaptic cell-adhesion protein, REEP3 with possible role in synaptic plasticity, calcium signaling, CTTNDP2 a G-protein-coupled serotonin receptor. PTPRD, a protein tyrosine phosphatase (PTP) family is also found. PTPs are known to be signaling molecule. ${ }^{5}$ Serotonin function in obsessive-compulsive disorder has been studied since the 80 's. Serotonin has thought to play a role in the pathophysiology of obsessive-compulsive disorder (OCD) because of the anti-obsessional effect of selective serotonin reuptake inhibitors (SSRIs). ${ }^{6}$ Medications such as tricyclic antidepressants (including clomipramine) and selective serotonin receptor inhibitors (SSRIs) are often used. Some of the more commonly used SSRIs are:Fluoxetine, Fluvoxamine, Paroxetine and Sertraline.

In addition to the use of serotonin reuptake medications, working with a psychologist who is trained in desensitization and cognitive behavioral therapy can be helpful. For example Exposure Response Prevention (ERP) therapy which involves patients trying to experience their triggers without doing their accompanying rituals - such as touching a toilet seat without washing their hands afterwards is used to teach them that nothing bad happens. According to the authors, findings signal potentially important facets of exposure for youth with OCD and have implications for treatment. A number of results also parallel recent findings in the adult literature, suggesting that there may be some continuity in exposure processes from child to adult development. $^{7}$

\section{Conclusion}

A meta-analysis of fourteen studies stated that severe OCD symptoms were consistently associated with greater sleep disturbance. While the sleep of OCD patients has not been a major focus to date, the existing literature suggests that addressing sleep disturbance in OCD patients may ensure a holistic approach to treatment, enhance treatment efficacy, mitigate relapse and protect against the onset of co-morbid psychiatric illnesses. ${ }^{8}$

\section{Acknowledgments}

No financial aid was received by the author in preparation of this manuscript and no Conflict of Interest! The author would like to thank Marvin H Berman PhD of Quietmind Foundation Elkins Park PA for suggestions and help in reviewing this manuscript.

\section{Conflicts of interest}

The author declares that there is no conflict of interest.

\section{References}

1. Peters, B. Effects of obsessive Compulsive Disorder (OCD) on sleep and insomnia: Does OCD commonly lead to difficulty sleeping. 2018.

2. Havnen A, Hovland A, Haug ET, et al. Sleep and heart rate variability in patients with obsessive -compulsive disorder; preliminary findings. Clin Neuropsych. 2013;10:56-60.

3. Apergis-Schoute AM, Gillan CM, Fineberg NA, et al. Neural basis of impaired safety signaling in Obsessive Compulsive Disorder. Proc Natl AcadSci U S A. 2017;114(12):3216-3221.

4. Hinds Al, Woody EZ, Van Ameringen M, et al. When Too Much Is Not Enough: Obsessive-Compulsive Disorder as Pathology of Stopping, Rather than Starting. PLoS ONE. 7(1):e30586.

5. Yue W, Cheng W, Liu Z, et al. Genome-wide DNA methylation analysis in obsessive-compulsive disorder patients. Proc Natl AcadSci U S A. 2017;14(12):3216-3221.

6. Charney DS, Goodman WK, Price LH, et al. Serotonin function in obsessive-compulsive disorder. A comparison of the effects of tryptophan and m-chlorophenylpiperazine in patients and healthy subjects. Arch Gen Psychiatry. 1988;45(2):177-185.

7. Kircanski K, Peris TS. Exposure and response prevention process predicts treatment outcome in youth with OCD. J Abnorm Child Psychol. 2015;43(3):543-552.

8. Paterson JL, Reynolds AC, Ferguson SA, et al. Sleep and obsessivecompulsive disorder (OCD). Sleep Med Rev. 2013;17(6):465-474. 